\title{
Point-of-care tobacco detection testing creates efficiency and reduces costs for employer-sponsored wellness programs
}

\section{Opinion}

Employer-sponsored wellness programs have become a common feature of population health management. Approximately 92 percent of employers with 200 or more employees currently offer some form of workplace wellness. ${ }^{1}$ Survey data indicates that one of the most frequently targeted behaviors is tobacco use, with 60percent of all employer-sponsored wellness programs offering screening and cessation activities. ${ }^{1}$ This is due in part to the average decrease in productivity and increase in health-related issues from employees that use tobacco products. These losses in productivity and increase in health expenditures can cost employers from $\$ 3400-\$ 5800$ dollars per employee per year. ${ }^{2}$ These factors make cotinine screening for tobacco product use, and providing cessation programs, not only beneficial to the employee's health, but also to their overall productivity. Conducting on-site screenings for tobacco use via cotinine can be difficult. The majority of point-of-care cotinine test for use in wellness screenings only offer qualitative results, utilizing saliva or urine as specimen sources. Saliva and urine methods make collection difficult and invasive to the employee, but also make it impossible to distinguish between passive exposure and more chronic tobacco use, without incurring further testing costs. This result in the need for venous blood draws to either confirm the presence of tobacco use, or as the initial test. Venous samples while highly accurate, require an outside reference laboratory for testing, often taking several days to receive the results. This route for screening for tobacco use via cotinine results misses the opportunity to immediately educate the employee about their tobacco use and cessation options.

The PTS Detect cotinine system, a quantitative point-of-care system manufactured by PTS Diagnostics, Indianapolis IN, was studied to determine its overall performance characteristics. The device is a lateral-flow, competitive immunoassay, used for the quantification of cotinine. This device was examined in a population of both nontobacco and tobacco users $(\mathrm{n}=49)$ to simulate the wellness program environment, and to determine if the device was able to properly place individuals into tobacco use categories when compared to the gold standard reference LC/MS-MS venous blood values. The category applied to the study for tobacco use was based upon a discriminatory cut-point for cotinine of $\geq 50 \mathrm{ng} / \mathrm{mL}$, which correlates with nicotine addiction. ${ }^{3}$ Each study participant had a venous draw for LC/MS-MS testing, and a capillary blood collection via fingerstick for testing on the point-of-care device. When compared to the LC/MS-MS method the POCT device demonstrated a specificity of $89 \%$ and a sensitivity of $100 \%$, demonstrating that the device can accurately and precisely determine an individual's smoking habits based upon quantitative cotinine levels.
Volume I Issue 3 - 2015

\author{
Lee B Springer \\ Colorado Technical University, Assistant Adjunct Professor, USA
}

Correspondence: Lee B Springer, Colorado Technical University, 4435 N Chestnut St Colorado Springs CO, 80907, Tel 6I29169732, Email navymedtech@hotmail.com

Received: October 25, 2015 | Published: October 28, 2015

Having the ability to effectively and immediately quantify an individual's cotinine level to determine tobacco use habits could potentially reduce the need for confirmatory testing. This aids employers by reducing the overall cost of providing employee wellness programs. Additional savings could also be achieved with a quantitative point-of-care cotinine test as it can immediately identifying employees that could benefit from tobacco cessation efforts, unlike traditional venous collection and testing. The POCT device's ability to effectively distinguish non-tobacco users, as well as accurately quantify an individual's tobacco use compared to LC/MSMS further demonstrates its ability to potentially eliminate the need for confirmatory testing. The device's demonstrated performance and features make it a unique solution for employer sponsored, or other types of wellness programs, as well as a viable substitute for venous blood collection for cotinine to screen for and determine tobacco use habits.

\section{Acknowledgements}

None.

\section{Conflict of interest}

The author declares no conflict of interest.

\section{References}

1. Mattke S, Schnyer Ch, Van Busum KR. A Review of the U.S. Workplace Wellness Market; 2012.

2. Berman M, Crane R, Seiber E, Munur M. Estimating the cost of a smoking employee. Tob Control. 2013;23(5):428-433.

3. Benowitz NL, Henningfield JE. Establishing a nicotine threshold for addiction. The implications for tobacco regulation. $N$ Engl $J$ Med. 1994;331(2):123-125. 